\title{
Extended Enterprise Collaboration for System-of- Systems Requirements Engineering: Challenges in the Era of COVID-19
}

\author{
Afef Awadid and Anouk Dubois \\ IRT SystemX, Palaiseau, France \\ \{afef.awadid, anouk. dubois\} @irt-systemx.fr
}

\begin{abstract}
Given the complexity and evolutionary nature of a System-of-Systems (SoS) entailing frequently changing goals and requirements, extended enterprise collaboration is needed. The idea behind extended enterprise collaboration consists in teaming up with other stakeholders in partnerships in order to manage the skills, risks and investments required to rush the product to market. However, collaboration is far from being trivial, as it is constrained by several challenges such as heterogeneity of stakeholders and their ways of working - let alone global crisis like COVID-19. Thus, an overview of these challenges from both academic and industrial perspectives would be beneficial. With this in mind, based on a survey, this paper provides a comprehensive overview that goes beyond the challenges, to consider the benefits of, methods for, barriers and bridges to efficient collaboration. The survey was conducted among research-active academics in the field of SoS engineering, and companies in the US and European aerospace and defense industries. On the one hand, the results show that in response to COVID19, work agreements (clear policies, commonly used processes and procedures, tools interoperability, clarity around roles and responsibilities of each collaborating firm, and proper change communication) are required in order to reduce uncertainty. On the other hand, it was revealed that "human bridges" are the backbone of extended enterprise collaboration. It is, indeed, human factors that either impede or promote efficient collaboration. An important finding that comes in agreement with recent calls for shifting the focus from "technology-centered" to "human-centered" SoS engineering.
\end{abstract}

Keywords: SoS Engineering, Requirements Engineering, Extended Enterprise Collaboration, COVID-19, Survey, Academics, Practitioners.

\section{Introduction}

By bringing to the fore health, food security, social care and transport considerations, the COVID-19 pandemic plainly shows that societal challenges cannot be parochial. Instead, they are global in nature [1], and hence beyond the capacity of any one sector, discipline, region, or country to handle them. Relying on extended enterprise collaboration, the area of Systems of Systems Engineering (SoSE) has a potential to overcome many of these societal challenges [2]. Contrarily to traditional Systems Engineering 
which emphasizes on a single system, SoSE focuses on a class of complex systems, whose constituents are themselves complex [3]. At the same time, SoSE is typically based on an extended enterprise collaboration, where different partner organizations/ stakeholders work together on the ground of a long-term agreement "to provide products and services in a well-defined yet evolving market segment" [4]. Consequently, at the core of an extended enterprise is a collaboration between multiple stakeholders referring to multidisciplinary teams from different organizations, standing to gain or lose from the success or failure of a System of System (SoS) [5].

However, although it may seem a straightforward working method, collaboration is far from being trivial. Rather, it is a key process that defines the way in which the stakeholders interact with each other, and that needs to be efficient [6]. An efficient collaboration is expected to be even more important in the future as unconventional solutions and disruptive technologies might be applied in order to develop sustainable and successful SoSs. Increasing the quality of the design process and reducing the leadtime are among the major reasons for which an efficient collaboration is asked for. Nevertheless, barriers such as heterogeneity of stakeholders and their ways of working [7] - let alone global crisis like COVID-19 pandemic impede such collaboration. These barriers have entailed serious challenges related to extended enterprise collaboration. One of them is the collaborative decision making (see e.g., [8] [9]). As we move towards a "Cross-organizational and cross-country integration of competences" [10], the idea of overcoming these challenges is increasingly being taken up in both academia and industry. However, what is still missing is an overview of these challenges from both academic and industrial perspectives. On the one hand, such an overview should consider the context of COVID-19, as this crisis has arguably influenced extended enterprise collaboration [11]. On the other hand, it will help set a new research agenda on extended enterprise collaboration for system-of-systems requirements engineering. Therefore, the time has come to provide a comprehensive overview that goes beyond the challenges, to consider the benefits of, methods and techniques for, and barriers and bridges to efficient collaboration.

To do so, this paper reports on a survey that addressed these issues. The survey was conducted among two categories of participants. First, eminent research active academics in the field of SoSE. Second, US and European companies in the aerospace and defense industry, as representatives of stakeholders. The purpose of this paper is to provide a global glance of what extended enterprise collaboration for system-of-systems requirements engineering is about (benefits, challenges, barriers and bridges), particularly in the era of COVID-19.

Following this introduction, the theoretical background and the methodological approach of this work are presented (section 2). Afterwards, the results of the study are disclosed (section 3). Subsequently, the obtained results are discussed (section 4). Finally, concluding remarks will round off the paper (section 5). 


\section{Theoretical Background and Methodological Approach}

We provide the background of this work in Section 2.1. Section 2.2 highlights the methodological approach used in this paper.

\subsection{Theoretical Background}

As mentioned earlier, a SoS is a complex system, which are in turn made up of complex constituent systems. An important point to be stressed out here is the significant difference between monolithic complex systems (e.g., a ship), and a SoS (e.g., a maritime search and rescue (SAR)). A SAR is considered as a SoS as the maritime search and rescue operations involve complex constituent systems like boats, helicopter, and aircrafts. To help distinguish a SoS from a monolithic complex system, [12] identifies five characteristics of a SoS. First, the operational independence of its constituent systems (i.e., each constituent system is capable of operating independently and achieving its own goals). Second, the managerial independence of its constituent systems (i.e., the constituent systems are managed independently so that they can be added or removed from the SoS). Third, the evolutionary nature of a SoS (i.e., the objectives of a SoS evolves over time to respond to the changing needs. Consequently, functions and purposes can be added, modified or removed as needed). Fourth, the emergent behavior of a SoS that cannot be bounded to any constituent system (i.e., the behavior and functionality of the SoS develop in a manner not achieved by the individual systems). Fifth, the geographical distribution of its constituent systems, which restricts their interactions to information exchange).

Owing to the complexity of a SoS and hence the complexity of its development requiring a breadth of skills and knowledge [13] so large that a single company cannot possess all the expertise themselves [14], the need for extended enterprise collaboration is unquestioned. Alternatively referred to as a "value chain" or a "supply chain", "the extended enterprise is a loosely coupled, self-organizing network of firms that combine their economic output to provide product and service offerings to the market" [15]. Building on this, the idea behind extended enterprise collaboration consists in teaming up with other stakeholders (e.g., customers, prime contractors, suppliers and government agencies) in partnerships in order to manage the skills, risks and investments required to rush the product to market. Extended enterprise collaboration is particularly crucial for SoS' requirements engineering. There are two reasons for this claim. First, "requirements engineering is a vital part of the SoS development lifecycle" [16]. Accordingly, the successful SoS development depends heavily on how well the underlying requirements are engineered. Second, due to the evolutionary nature of a SoS entailing frequently changing goals and needs, SoS' requirements engineering is a challenging activity. It is from these reasons that comes the motivation for this work.

\subsection{Methodological Approach}

Because it is useful for unearthing context-specific insights and obtaining a clear understanding of a real-life situation [17] [18], a qualitative research approach is employed in order to achieve the purpose of this paper. An anonymous survey along with a cover letter explaining the study was mailed in March 2020 to a random sample of 
(1) 39 professors in the field of systems engineering and (2) 13 companies active as first tier suppliers in the US and European aerospace and defense industries. The reason for using a survey method was to be able to reach a wider audience of respondents knowledgeable in the extended enterprise collaboration for SoS' requirement engineering. Of 52 surveys mailed, 34 were completed and returned ( 28 academics and six practitioners answered to the survey), for a total response rate of $65.38 \%$. The industrial respondents were identified with the help of gatekeepers [19] within each company. These gatekeepers are cognizant of who had expertise and experience in the field of enterprise collaboration.

For confidential reasons and as part of anonymizing the data, the identities of the respondents and the companies were withheld. The survey consists of five questions, with free text answer options:

1. In your opinion, what are the benefits of efficient collaboration among stakeholders for SoS requirements engineering?

2. What are the challenges of extended enterprise collaboration, including those related to COVID-19?

3. What barriers actually hinder efficient collaboration among stakeholders?

4. Which bridges/ factors might mitigate the potential barriers?

5. Which methods, techniques and/or tools do you think help to achieve an efficient collaboration?

The first question seeks to uncover the why behind efficient enterprise collaboration. The second question aims to highlight the key challenges pertaining to extended enterprise collaboration for SoS' requirements engineering, considering the context of COVID 19 outbreak. Identifying the barriers obstructing the efficient collaboration is the purpose of the third question. The fourth one pursues to reveal the factors that might facilitate the desired collaboration. Finally, the focus of the fifth question is on the methods and tools that might support the achievement of efficient collaboration.

\section{$3 \quad$ Results}

By addressing the above questions, this section exhibits the results of the survey and their analysis.

\subsection{Benefits of Extended Enterprise Collaboration}

Before revealing the benefits of extended enterprise collaboration as perceived by academics and practitioners, it is worth noting that both groups of respondents recognize the criticality of such a collaboration for SoS' requirements engineering. They justify this by highlighting the multiple opportunities it provides. The respondents' answers to the question about the benefits of efficient enterprise collaboration diverge greatly from a simple "time advantages" to more elaborate answers such as "Accelerating SoS development, and hence reducing the lead-time". Some respondents transcend the simple identification of collaboration benefits, to elucidate how they can be achieved. Still, the 
different answers to this first question can be summarized in four points that are confirmed, sometimes with varying degrees of intensity, by both respondents' groups.

First, reducing SoS development costs. From the industrial standpoint, a SoS requires large investments to be developed. Based on revenue sharing partnerships, an extended enterprise collaboration allows to efficiently manage these investments. Second, accelerating SoS development, referring to reducing SoS development time, and hence reducing the lead-time. Out of the total respondents, almost $61 \%$ admit that 24 hour co-working combining people from different time zones allows to accelerate SoS development. Consequently, interesting is the mutual focus by both groups of respondents (academics and practitioners) on the opportunities of cross-country/ external collaboration. Third, improving the overall quality of the SoS. Although both groups acknowledge the importance of efficient enterprise collaboration for SoS' requirements engineering in enhancing the overall quality of the SoS to be developped, there is no common agreement on how a high-quality SoS should look like.

From an academic standpoint, a high-quality SoS is a working and meaningfull SoS. Against this background, it is stated commonly that efficient enterprise collaboration plays a critical role in providing such a SoS, as it allows to get relevant and proper requirements. Whereas, from an industrial standpoint, a high-quality $\mathrm{SoS}$ is the one that keeps up with the requirements of the customer. Pursuant to this standpoint, extended enterprise collaboration has made possible the delivery of such a SoS, by fostering a continuous voice of the customer, and a continuous optimization of value. Fourth, promoting innovation. As a matter of fact, some of the respondents from both groups consider that extended enterprise collaboration for SoS' requirements engineering, and more specifically, cross-country collaboration brings multiple perspectives and diversity on ideas, and hence promotes innovation. Table 1 exhibits these four benefits, along with respondents' percent distribution by group.

Table 1. Benefits of enterprise collaboration from academic and industrial standpoints.

\begin{tabular}{|c|c|c|}
\hline $\begin{array}{ll}\text { Benefits } & \text { Respondent groups } \\
\end{array}$ & Academics & Practitioners \\
\hline Reducing SoS development costs & $100 \%(28$ out of 28$)$ & $100 \%(6$ out of 6$)$ \\
\hline Accelerating SoS development & $100 \%$ & $100 \%$ \\
\hline Improving the overall quality of the SoS & $82.14 \%(23$ out of 28$)$ & $100 \%$ \\
\hline Promoting innovation & $10.71 \%(3$ out of 28$)$ & $66.66 \%(4$ out of 6$)$ \\
\hline
\end{tabular}

\subsection{Challenges of Extended Enterprise Collaboration}

A closer look at the total answers of all respondents to the question about the challenges of extended enterprise collaboration, allows to classify these challenges into general and specific. Unlike general challenges, specific ones are related to a particular context (COVID-19 pandemic and/ or external collaboration).

In total, five general challenges are shared between both respondent groups. First, the varying expectations and / or goals of the stakeholders, on account of the heterogeneity of their requirements for the SoS. There are, indeed, stakeholders at two levels: the SoS and the constituent systems levels. Each stakeholder group has its own goals with respect to the SoS. Consequently, two types of requirements usually defined in 
terms of capabilities exist: (i) SoS-level requirements referring to properties of the overall SoS, and (ii) constituent system-level requirements which are assigned to particular individual systems (s). Collectively, this creates a complex stakeholder environment, in which conflicting requirements and contradictory goals are most likely to occur. This is very challenging for requirements elicitation, management and documentation, especially with respect to consistency and communication.

Second, the difficulty in knowledge sharing. Out of 28 academic respondents, 16 (57.14\%) attribute this difficulty to the distribution of data storage across different organizations and confidentiality issues (access federation). This distribution complicates the search for and the retrieval of information across organizational borders. With this in mind, extended enterprise collaboration may be ineffective if the IT resources of each partner organization are not mutually accessible. However, some of the practitioner respondents relate the difficulty in knowledge sharing to the fear of losing competitive advantage. Third, the heterogeneity of SoS engineering tools used by the different stakeholders, making it difficult to render them compatible.

Fourth, the lack of understanding among the stakeholders. As reported by both academics and practitioners, this lack of understanding results from the multidisciplinarity of stakeholders involved. Because of this multidisciplinarity, a broader understanding and a commonly agreed definition of SoS are not that easy to achieve, let alone jointly agreed requirements. Fifth, the complexity of collaborative-decision making. More than $50 \%$ of all respondents argued that the variety of stakeholders and their interests, leading often to contrasting interpretations and contradictory goals, makes it very difficult to reach a collegial decision on what requirements to validate, to prioritize and most importantly why.

In addition to the five aforementioned challenges, some practitioners (4 out of 6) drew attention to another one - the lack of trust among stakeholders leading to missing openness and transparency. Behind this lack of trust is the fear of losing competitive advantage. In this vein, intellectual property rights and evaluations of what knowledge to exchange with partners and what not to exchange are brought forward as serious issues. From a focal company perspective, it is therefore essential to strike a balance between not disclosing too much, but still enough to deliver value to the partners and the customers, that is, to provide a high-quality SoS. Fig. 1 and Fig. 2 display these general challenges and their corresponding respondents' percentages respectively according to academics and practitioners. 


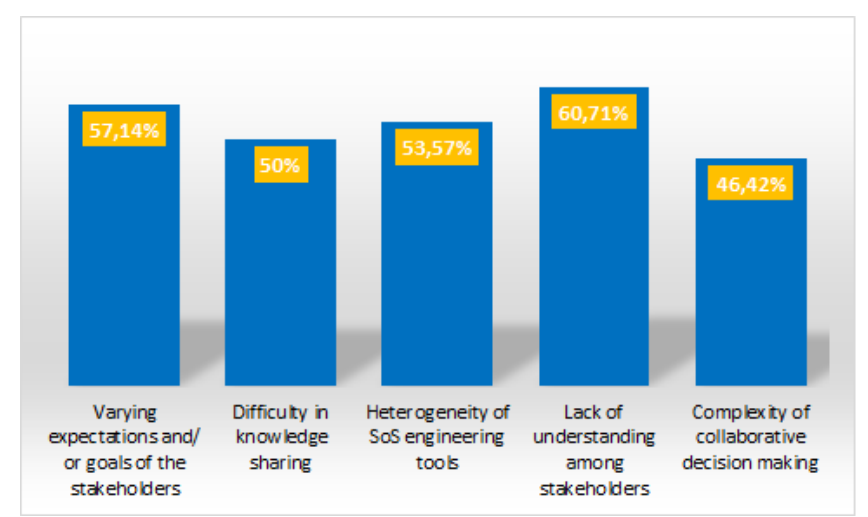

Fig. 1. General challenges of extended enterprise collaboration from an academic standpoint.

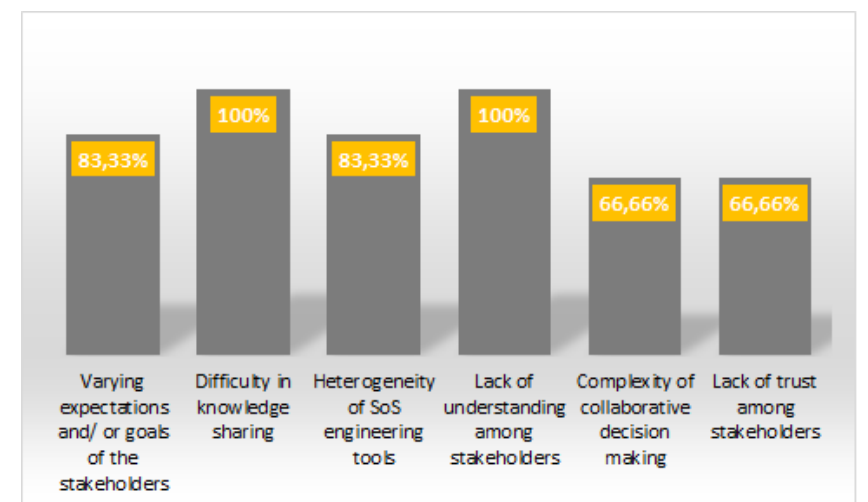

Fig. 2. General challenges of extended enterprise collaboration from an industrial standpoint.

As pointed out at the beginning of this subsection, the specific challenges of extended enterprise collaboration are those related to a particular context (the external collaboration and/ or the COVID-19 pandemic).

Based on the answers given by both academics and industrials, three main challenges tied to external collaboration can be derived. The first challenge is the time difference. In fact, according to some respondents (one practitioner and six academics, accounting for a total percentage of $20.58 \%$ ), different time zones lead to a difficulty in coordinating work, and hence to a small "window of opportunity" for synchronous collaboration. The second challenge is the cultural differences. The latter is raised by three practitioners and eleven academics, combining for a total percent cover of $41.17 \%$. Some of the academic respondents spoke of the so-called "Radius of collaborative colocation", meaning that people distant geographically and/ or culturally are less likely to collaborate, particularly in a non-planned manner. The third challenge is the differences in regulation, for example General Data Protection Regulation (GDPR) in Europe vs regulations (or lack thereof) in other parts of the world. 
When it comes to the challenges pertaining to COVID-19, a consensus is observed between academics and practitioners. These shared challenges can be summarized as follows:

- Difficulty in replacing on-site meetings with online ones: the strict measures implemented by the large companies make personal meetings difficult. This is very challenging when intricate problems need to be discussed. This challenge is experienced by two practitioners from the defense industry, who collaborate internally. Referring to their answers, the required interactions are complex and difficult to achieve without personal contact.

- Difficulty in working with people you have never met: This challenge was recognized as a critical issue by both groups of respondents (three practitioners and nineteen academics, representing a total percentage of 64.7\%). In line with this, one of the respondents stated "I worked from home for the better part of 15 years, but I had worked with my team face-to-face for years before that. Zoom meetings are not the same as face-to-face, and can be awkward with people who you don't know at all."

- Increased uncertainty in the extended enterprise collaboration process: About $71 \%$ of the total respondents assume that the COVID-19 pandemic has undoubtedly changed generic organizational aspects such as policy, security, technical means, and relation with suppliers.

- Difficulty in making decisions under pressure (the risk of an economic crisis) in an uncertain context. Many are the respondents (more than 60\%) who asserted that stress and anxiety caused by the pandemic has certainly affected the ability to process and make decisions under pressure.

- Potential disruption of the extended enterprise collaboration process. Out of 34 respondents, 9 (one practitioner and eight academics, amounting to a total percentage of $26.47 \%$ ) claimed that changing policies and strategies in response to COVID-19 pandemic, along with the difference between the stakeholders' approaches in dealing with uncertainties are prone to trigger disruption or role conflicts in the collaboration.

\subsection{Barriers to Efficient Enterprise Collaboration}

It is worth noting that a multitude of barriers to efficient enterprise collaboration were enumerated by both academics and industrials. Nevertheless, these barriers can be sorted into four major categories: human, organizational, technical and contextual barriers.

1. Human barriers: Surprisingly, more than $80 \%$ of practitioners (5 out of 6 ) and about $79 \%$ of academics ( 22 out of 28 ) sharpen the focus on human barriers. The latter include among others the high operational tempo of stakeholders. More than 6 in 10 respondents said the op tempo resulted in unhealthy stress levels, affecting the performance of collaborators, and hence making it difficult for them to achieve the desired goals, the lack of trust, openness and transparency among stakeholders, the absence of strong leadership. According to some practitioners, it becomes over- 
whelmingly apparent that in the absence of strong lead engineer, there is either immature or no systems engineering at all, the lack of common interest among stakeholders, communication problems and misunderstandings, leading to SoS' requirements elicitation difficulties, the differences in applied nomenclature and languages between the involved disciplines. In this regard, the "not invented here" syndrome is seen as a cause of aversion to changes and a lack of trust, the lack of knowledge sharing (leading to unnecessary double actions, repeated errors, and overlapping results).

2. Organizational barriers: By referring to the answers of all respondents, organizational barriers are mainly due to managerial complexity as well as property and resource protection. Non-aligned strategies, lack of overarching and centralized management, political choices, inflexibility towards changes, operating procedures and regulations are among the key factors causing the managerial complexity. In addition, the measures resulting from extensive security policies implemented by most organizations in order to protect intellectual and resources property make the exchange of information among stakeholders a complex and time-consuming task.

3. Technical barriers: Many are the technical barriers that were shared between academics and industrials. In the following, some examples of these barriers are given. The heterogeneity of tools and computing infrastructures, the licensing issues preventing use of an organization's computational resources or commercial tools by others, the lack of appropriate information systems for organizing the activities and data in a collaborative set-up, the complex security policies (resulting from rigid security measures), the dynamic organizational and IT - environments leading to difficulties in ensuring continuous working method/ solution, the lack of trust in the wholeness of available resources and information.

4. Contextual barriers: What is interesting to note here is that these barriers refer to those related to contextual circumstances (e.g., public health threats, like COVID19). They are, therefore, high-level barriers that may induce in turn human, organizational and/ or technical barriers. Respondents' answers that can fit this category include increased level of uncertainty, difficulty in digitally collaborating, and difficulty in making orientation/meet-and-greet trips, difficulty in decision making, delay and extra costs to accommodate the change.

\subsection{Bridges to Efficient Enterprise Collaboration}

Although they may seem quite heterogeneous at a first glance, the respondents' answers to the question about the 'bridges to efficient enterprise collaboration' cover only three levels: human, organizational and technical. They are, therefore, in congruence with the answers to the previous question. It is noteworthy that 'human bridges' were recognized as the most popular factors that might mitigate the collaboration barriers ( 5 practitioners and 24 academics, representing a total percentage of $85.29 \%$ ), against $64.7 \%$ for 'technical bridges' and $55.88 \%$ for organizational ones. In the following, the three categories of bridges along with specific examples of each category, based on the responses of the survey participants: 
Human Bridges. This category includes:

- Clarity around roles and responsibilities of each collaborating firm in order to reduce uncertainty and ambiguity in the collaboration process.

- Copious communications and collaboration among the stakeholders, especially in managing the requirements.

- Trust and a high level of liability among the stakeholders.

- Autonomous translation of used semantics.

- Autonomous translation between used standards from various domains.

- Technical Leadership Competencies for both technical and enabling competencies

Organizational Bridges. It contains the following organizational factors:

- Schedules and budgets alignment.

- Proper change communication: If one organization is ready to deploy, but another part of the SoS is not ready or they made a change which was not communicated properly, then the first organization may have to delay and incur extra costs to accommodate the change.

- Overall baseline for the whole SoS system, under strict configuration control.

- Agreement on commonly used processes and tools.

- Common values regarding the SoS to be developed.

- Consistent "Systems Thinking" engagements.

- Work agreements (clear policies, procedures and approaches) to reduce ambiguity and uncertainty.

Technical Bridges. Examples of technical factors found in this category are:

- Computing infrastructures and tools Interoperability.

- Adequate information systems development for a collaborative set-up.

- Reference architectures and reference platform implementations.

- Sound Systems Engineering methodologies implementation.

- Non-synchronous communication tools.

- IEEE 15288 System and Software Engineering Processes.

\subsection{Methods, Techniques and or/ Tools for Achieving Efficient Collaboration}

The answers to the question about the methods, techniques and/ or tools for achieving efficient collaboration can be divided into two categories: approaches and tools. In the first category, one can find as examples of approaches suggested by the respondents to the survey:

- Approaches for reducing the complexity. Many of the respondents argued that there is no "one best way". Instead, different settings require different approaches.

- Applying "just enough" process to not over burden.

- Using transparent processes.

- Prioritized, integrated backlog.

- Excellent communications, maintaining configuration control over the SoS baseline (if one exists), staying in touch with the other developing organizations. 
- Using Model-Based Systems Engineering (MBSE) approach as much as possible to alleviate the document dependency issues, and doing a lot of up front development planning among the organizations.

- Adopting a sound systems engineering methodology would help, even for SoS.

The second categories of answers put emphasis on the tools, and includes among others the following suggestions:

- Using Arrowhead Framework (now becoming to Eclipse Arrowhead) as architecture and implementation platform. It provides translation concept and support for protocol translation.

- Using commonly agreed and easy to use tools.

- Using Wiki tools integrated with repositories.

\section{Discussion}

Looking at the results of this study, it is evident that extended enterprise collaboration is full of opportunities as well as challenges. Consequently, these results provide a roadmap that indicates what opportunities to maximize and what challenges to manage. Against this background, the answers to the questions about challenges and barriers complement each other, so that an overall picture of what hinder enterprise collaboration is given. Depending on the type of collaboration (internal or external), and considering the COVID-19 pandemic, these challenges are divided into two categories: general and specific. An emphasis on the specific ones shows that COVID-19 pandemic has affected both internal collaboration (manifested e.g., by a difficulty with online meetings) and external collaboration (revealed by the disruption of the collaboration process and hence the supply chain network).

Because of the COVID-19 crisis, the level of uncertainty becomes even higher and many of issues are even more critical to address. The biggest one is the heterogeneity of stakeholders (developing/governing organizations) supporting the SoS that have to collaborate in the requirements space. Who "owns" the SoS baseline? How is that communicated and maintained among all the different organizations? When one organization makes a change to their baseline, how is that communicated to the other organizations and how is that flowed through the requirements? Who controls configuration management of the SoS baseline? These are samples of intricate problems that need to be discussed face-to-face. Because of COVID-19 outbreak, this is no longer possible. In response to COVID-19 pandemic, it should be pointed out that work agreements (clear policies, commonly used processes, tools, approaches, clarity around roles and responsibilities of each collaborating firm, proper change communication, ...) are required in order to reduce ambiguity and uncertainty.

Albeit the multitude of barriers to efficient enterprise collaboration as perceived by academics and practitioners, this study allowed to classify them into four categories: human, organizational, technical and contextual. Examining the results with respect to these categories, it is apparent that "human bridges" are the backbone of extended en- 
terprise collaboration. It is, indeed, human factors that either impede or promote efficient collaboration. An interesting finding that comes in agreement with recent calls for shifting the focus from "technology-centered" to "human-centered" SoS engineering [20].

\section{$5 \quad$ Concluding Remarks}

By reporting on a survey, this paper provides an overview of what extended enterprise collaboration for SoS' requirements engineering is about (benefits, challenges, barriers and bridges), particularly in the era of COVID-19. The survey was conducted among research-active academics in the field of System-of-Systems engineering, and companies in the US and European aerospace and defense industries. The findings of this study are multifold. (i) Depending on the type of collaboration (internal or external) and considering the covid-19 pandemic, challenges of extended enterprise collaboration can be divided into general and specific. While existing barriers can be classified as human, organizational, technical or contextual.

(ii) In response to COVID-19, work agreements (clear policies, commonly used processes and procedures, tools interoperability, clarity around roles and responsibilities of each collaborating firm, and proper change communication) are required in order to reduce uncertainty. (iii) "Human bridges" are the backbone of extended enterprise collaboration. It is, indeed, human factors that either impede or promote efficient collaboration. An interesting finding that comes in agreement with recent calls for shifting the focus from "technology-centered" to "human-centered" SoS engineering. (iii) Digital collaboration cannot in any case replace/ be as efficient as on-site collaboration.

Despite the limitations of a survey of a small number of respondents, the chosen participants not only have in depth knowledge of the topic, but also have experienced the challenges either as practitioners or as research-active academics. By providing a roadmap indicating what opportunities to maximize and what challenges to manage, this paper will help set a new research agenda on extended enterprise collaboration for SoS' requirements engineering. Building on this, future research will address each challenge separately in depth, by investigating and evaluating the ability of artificial intelligence techniques in solving these challenges.

\section{References}

1. Dutta, S., Bilbao-Osorio, B.: The Global Information Technology report. Living in a Hyperconnected World, World Economic Forum (2012).

2. Maier, M.W.: Architecting Principles for Systems-of-Systems. Systems Engineering 1(4), 267-284 (1998).

3. Jamshidi, M.: System of Systems Engineering: Innovations for the Twenty-First Century. Wiley (2011). 
4. López-Ortega, O., de la Cruz, K. L.: Usage of Agent Technology to Coordinate Data Exchange in the Extended Enterprise. In Advanced Design and Manufacturing Based on STEP. Springer, London (2009).

5. Nuseibeh, B., Easterbrook, S.: Requirements engineering: a roadmap. In: 22nd International Conference on Software Engineering, Future of Software Engineering Track, pp. 35 - 46, Limerick, Ireland (2000).

6. Baalbergen, E. H., Moerland, E., Lammen, W. F., Ciampa, P. D.: Methods supporting the efficient collaborative design of future aircraft. In: CEAS Conference, Bucharest, Romania (2017).

7. Dumitrascu-Baldau, I., Dumitrascu, D. D.: A Systemic View of Interna-tional Projects with Virtual Teams. In: International conference on Knowledge-based organization, pp. 289-295, Sciendo (2018).

8. Bartelt, V. L., Urbaczewski, A., Mueller, A. G., Sarker, S.: Enabling collaboration and innovation in Denver's smart city through a living lab: a social capital perspective. European Journal of Information Systems, pp. 1-19 (2020).

9. Antoni, N., Giannopapa, C., Schrogl, K. U.: Legal and Policy Perspectives on Civil-Military Cooperation for the Establishment of Space Traffic Management. Space Policy (2020).

10. Ciampa, P. D., Moerland, E., Seider, D., Baalbergen, E., Lombardi, R., D'Ippolito, R.: A collaborative architecture supporting AGILE design of complex aeronautics products. In: 18th AIAA/ISSMO Multidisciplinary Analysis and Optimization Conference, p. 4138 (2017).

11. Golan, M. S., Jernegan, L. H., Linkov, I.: Trends and applications of resilience analytics in supply chain modeling: systematic literature review in the context of the COVID-19 pandemic. Environment Systems \& Decisions (2020).

12. Ncube, C., Lim, S. L., Dogan, H.: Identifying top challenges for international research on requirements engineering for systems of systems engineering. In: 21st IEEE International Requirements Engineering Conference, pp. 342-344. IEEE (2013).

13. Acha, V., Davies, A., Hobday, M., Salter, A.: Exploring the capital goods economy: complex product systems in the UK. Industrial and Corporate Change13(3), pp505-529 (2004).

14. Boardman, J.T., Clegg, B.T.: Structured engagement in the extended enterprise. International Journal of Operations \& Production Management 21(5-6), pp795-811 (2001).

15. Farrell, J. P. : What is the extended enterprise? Available at: https://jpfarrell.blogspot.com/2008/04/extended-enterprise.html (2008).

16. Andrews, Z., Ingram, C., Payne, R., Romanovsky, A., Holt, J., Perry, S.: Traceable Engineering of Fault-Tolerant SoSs. In INCOSE International Symposium, pp. 258-273 (2014).

17. Aberdeen, T., Yin, RK.: Case study research: Design and methods. Thousand Oaks, CA: Sage. The Canadian Journal of Action Research 14(1), pp. 69-71 (2013).

18. Venkatesh, V., Brown, S. A., Bala, H.: Bridging the qualitative-quantitative divide: Guidelines for conducting mixed methods research in information systems. MIS Quarterly 37(1), pp. 21-54 (2013).

19. Neyland, D.: Organizational Ethnography. Sage Publications, London, United Kingdom (2007).

20. Boy, G. A.: Human-Systems Integration: From Virtual to Tangible. CRC Press (2020). 